\title{
Design of A Propulsion Cycle for Endless Sliding on Frictional Ground Using Rotating Masses
}

\author{
Christopher Provatidis \\ Department of Mechanical Engineering, National Technical University of Athens, Greece \\ *Corresponding Author: cprovat@central.ntua.gr
}

Copyright (C) 2014 Horizon Research Publishing. All rights reserved.

\begin{abstract}
This paper proposes a propulsion cycle to enable an object to perform slow unidirectional sliding on the horizontal ground when an inertial drive, made of contra-rotating eccentric masses, is attached to it. Two masses are driven by one or two synchronized motors, which rotate at a variable angular velocity according to a predefined function with a period of 360 degrees. The aforementioned predefined law of rotation constitutes the proposed propulsion cycle, which when repeatedly followed, allows for an endless forward sliding of the object. It was found that the maximum static friction between the object and the ground is the key factor that offers repeated support for the object to develop linear momentum in the beginning of every new cycle. The simulation is based on closed form analytical solutions of the ordinary differential equations for the unidirectional motion of the object to which the rotating masses are attached.
\end{abstract}

Keywords Inertial Drive, Rotation, Locomotion, Guidance, Perpetual motion

\section{Introduction}

The usefulness of inertial propulsion is well known since the ancient times when Greek athletes used to rotate halteres (dumbbells) in order to increase their performance in the long jump [1, 2]. Today, halteres are used as biologically-inspired sensors [3]. More generally, rotating eccentric masses are used in many engineering applications such as actuators for the motion of microrobotic platforms [4], toys, self-synchronous vibrating feeders, synchronous vibrating conveyors, self-synchronous probability screens, synchronous vibrating coolers [5], including the well-known mobile phone shakers (buzzers).

In addition, rotating masses have been claimed to be capable of producing vehicle propulsion as well. One of the first inertial propulsion drives of last century was patented in 1933 by the late Italian university professor Marco Todeschini [6], while a second one was patented in 1959 by
Norman Dean (a civil service employee residing in Washington DC). Dean applied in-plane contra-rotated eccentric masses (today his concept is known as "Dean drive") [7] and he claimed for reactionless propulsion; his drive became a matter of debate but it also inspired hundreds of new patents [8, pp. 249-261].

From a textbook of Blekhman (Russian Academy of Sciences), which was translated in English in 2000, we learn that the "notorious" Dean drive has become a matter of past research in Soviet Union [9, p.15 and p.19; 10]. Surprisingly, today relevant research continues and one can easily find Internet and press reports concerning claimed successful perpetual motion machines in Russia (see for example [11]); at the same time there are many relevant Internet articles referring to "pseudoscience". Although details for the technology applied are not available, in order to resolve confusion still existing, the detailed mechanics of Dean drive have been recently investigated for the vertical motion of an object [12, 13], while its elastodynamics and equivalent electromagnetic analogues have been also studied [14]. The possibility of replacing rockets [15] through novel drives to achieve space propulsion (called "alternative propulsion"), seems to be a matter of scientific and technological prestige for many countries including China [16]. The interested reader can explore a number of Internet sources concerning, for example, "inertial propulsion" (a term already scientifically established [17]), "inertial drive" (or "inertiod"), "antigravity propulsion", "torsion field" and many others.

The rationale is as follows. Since experimental vehicles (object plus the attached inertial drive) move without expelling a reaction mass and since no aerodynamics phenomena appear (motion occurs even if the drive operates within a sealed closed box), the basic question that arises concerns the cause of the motion (for example, [18] refers to built-in wings). A qualitative answer has been given by Millis [8, pp.250-251], who has attributed the net motion "to the connection to the ground, and particularly to the difference between the static friction (sometimes called sticktion) and the dynamic friction between the device and the ground that is required for their operation". 
Within the above context, this paper aims at quantitatively investigating the conditions under which a contra-rotating drive may cause slow motion of the vehicle (or object) to which it is attached, on the frictional horizontal ground. The study proposes an indicative design of motor guidance, according to which the eccentric masses rotate with a time dependent speed that ensures forward sliding of the vehicle, with a period of 360 degrees.

\section{Inertial Drive}

\subsection{Description}

There are many types of inertial drives, which are based either on gyroscopes or on nearly concentrated rotating masses. Between them we can distinguish the so-called "Dean drive" [7]. The latter is a device that consists of two exciters (rotating masses, No.1 and No.2), attached to an object (vehicle) of centroid G, as schematically is shown in Fig. 1. Below we present the case according to which the rods and the masses rotate on the horizontal $x y$-plane on which the object is allowed to slide. The two masses are assumed to be fully synchronized so as to possess identical angular velocities in magnitude but opposite in direction. Due to the contra-rotation, it is evident that the potential motion of the object will take place entirely along the direction of $y$-axis.

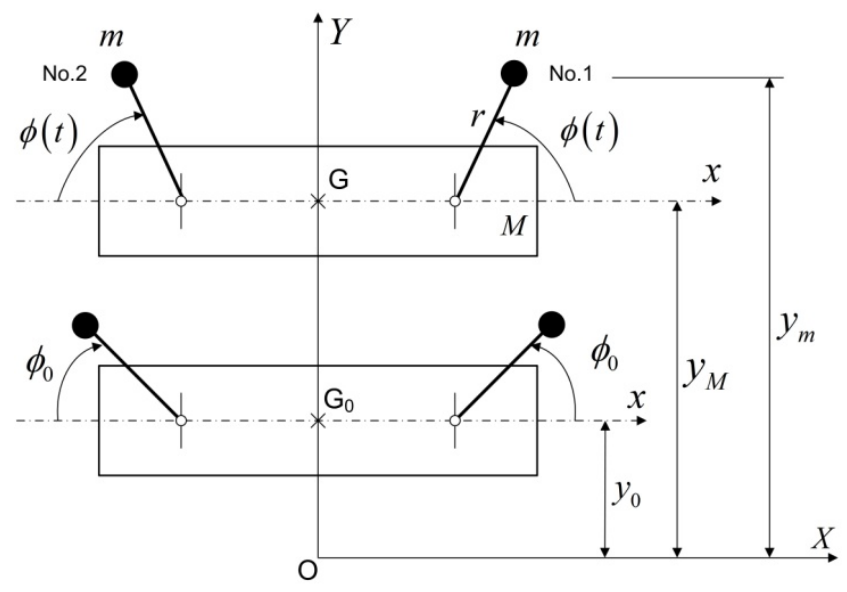

Figure 1. Top view of the drive on the ground.

\subsection{Equations of Motion}

Referring to Fig. 1, let us denote $y_{M}$ the position of the object (of mass $M$ ), $y_{m}$ the position of every eccentric (of mass $m$ ), and $\phi(t)$ the time varying polar angle formed by the $x$-axis and the rotating rod. In the beginning of time $(t=0)$, at which the centroid of the object is at the point $\mathrm{G}_{0}$ while the rods form the angle $\phi_{0}$, as shown in the bottom of Fig. 1, the object is at rest and suddenly is released to move on the ground. Obviously, the motion of the object will be possible only if the inertial force overcomes the maximum static friction, as will be explained later on.

Due to the fact that the contra-rotation of the two rotating masses cancels the two $x$-components of the inertial forces, their resultant will appear only in the $y$-direction and will be equal to $-2 m \ddot{y}_{m}$. The external forces on the object (of mass $M$ ) consist of (i) the aforementioned d'Alembert force $\left(-2 m \ddot{y}_{m}\right)$ plus (ii) the friction force $F_{f}$ that is directed opposite to the velocity $\dot{y}_{M}$. Therefore, Newton's second law becomes:

$$
M \ddot{y}_{M}=-2 m \ddot{y}_{m}-F_{f}
$$

Also, the relationship between $y_{m}$ and $y_{M}$ is (see Fig. 1):

$$
y_{m}=y_{M}+r \sin \phi
$$

Considering $\omega=\dot{\phi}$, by virtue of (2), $\ddot{y}_{m}$ is given by:

$$
\ddot{y}_{m}=\ddot{y}_{M}+r\left(-\omega^{2} \sin \phi+\dot{\omega} \cos \phi\right)
$$

Substituting (3) into (1), one obtains

$$
(2 m+M) \ddot{y}_{M}=\underbrace{2 m r\left(\omega^{2} \sin \phi-\dot{\omega} \cos \phi\right)}_{F_{\text {inertial }}}-F_{f}
$$

Dividing both parts of (4) by $(2 m+M)$ and introducing the parameter

$$
\lambda=\frac{2 m r}{(2 m+M)},
$$

the former leads to the final equation of motion:

$$
\ddot{y}_{M}=\lambda\left(\omega^{2} \sin \phi-\dot{\omega} \cos \phi\right)-F_{f} /(2 m+M),
$$

whereas the total inertial force is given by:

$$
\begin{aligned}
F_{\text {inertial }} & =2 m r\left(\omega^{2} \sin \phi-\dot{\omega} \cos \phi\right) \\
& =(2 m+M) \lambda\left(\omega^{2} \sin \phi-\dot{\omega} \cos \phi\right)
\end{aligned}
$$

\subsection{Friction}

\subsubsection{Definitions}

In terms of the normal force $N$, the static friction is written as

$$
F_{s} \leq F_{s, \max } \equiv \mu_{s} N,
$$

while the kinetic friction as

$$
F_{k}=\mu_{k} N \text {, }
$$

where $\mu_{s}$ and $\mu_{k}$ is the coefficient of static and kinetic friction, respectively. In (8) and (9) we recognize the static friction $\left(F_{s}\right)$ and its maximum value $\left(F_{s, \max }\right)$, as well as 
the kinetic friction $\left(F_{k}\right)$ of constant value.

Usually, for a given pair of surfaces:

$$
\mu_{s}>\mu_{k} \text {. }
$$

\subsubsection{Friction models}

When the object moves, the induced friction equals to the kinetic one (of a constant value). In contrast, when the velocity vanishes (object temporarily at rest) there are two alternative cases depending on the instantaneous acceleration:

- If the inertial force is smaller than the maximum static value the object does not tend to slide (therefore, the friction equals to the inertial force and the acceleration vanishes $\left(\ddot{y}_{M}=0\right)$.

- If the inertial force is greater than the maximum static value, the object tends to slide (therefore, the friction equals to the kinetic friction and the object is accelerated $\left(\ddot{y}_{M} \neq 0\right)$.

The aforementioned model can be easily described using the unified formula:

$$
F_{f}=\left\{\begin{array}{ll}
F_{k} \operatorname{sgn}\left(\dot{y}_{M}\right), & \dot{y}_{M} \neq 0 \\
F_{\text {inertial }}, & \left\|F_{\text {inertial }}\right\|<F_{s, \text { max }}, \\
\mu_{s} N \cdot \operatorname{sgn}\left(F_{\text {inertial }}\right), & \left\|F_{\text {inertial }}\right\|>F_{s, \text { max }}
\end{array},\right.
$$

where the function $\operatorname{sgn}\left(\dot{y}_{M}\right)$ is defined by:

$$
\operatorname{sgn}\left(\dot{y}_{M}\right)= \begin{cases}+1, & \dot{y}_{M}>0 \\ 0, & \dot{y}_{M}=0 \\ -1, & \dot{y}_{M}<0\end{cases}
$$

In our case, the normal force is permanently given by

$$
N=(2 m+M) g \text {, }
$$

and therefore, if the combination $\left(\dot{y}_{M}=\ddot{y}_{M}=0\right)$ never occurs, (6) becomes:

$$
\ddot{y}_{M}=\lambda\left(\omega^{2} \sin \phi-\dot{\omega} \cos \phi\right)-\mu_{k} g \cdot \operatorname{sgn}\left(\dot{y}_{M}\right) .
$$

\section{Design Guidelines of an Inertial Cycle}

\subsection{General}

We aim at determining a function of motor shaft's angular velocity versus polar angle or time, i.e. $\omega(\phi)$ or $\omega(t)$, which is periodic in $\phi \in[0,2 \pi]$ thus it is continuously repeated every 360 degrees of rotation.

The requirements for the design are as follows:

- The vehicle is initially at rest

- Only forward motion is desired

- The cycle is always the same.
One design restriction is that the selection of a high angular velocity, $\omega$, may lead to a high inertial force. If the latter exceeds the maximum static friction, will cause sliding that obviously will be reversed every 180 degrees of rotation. Therefore, since we wish only a forward motion of the vehicle, the best design solution is to start the rotation of the masses from a zero angular velocity $\left(\omega_{i}=0\right)$ and, after 360 degrees, the angular velocity returns again to the zero value $\left(\omega_{e}=0\right)$, as shown in Fig. 2. If we also achieve that the object (vehicle) has, at both the beginning ('i') and the end ('e') of every cycle, a zero linear velocity $\left(v_{0}=0\right)$, then we have found an accepted design solution.

The proposed design solution consists of three successive phases of rotation (shown in Fig. 2) as follows:

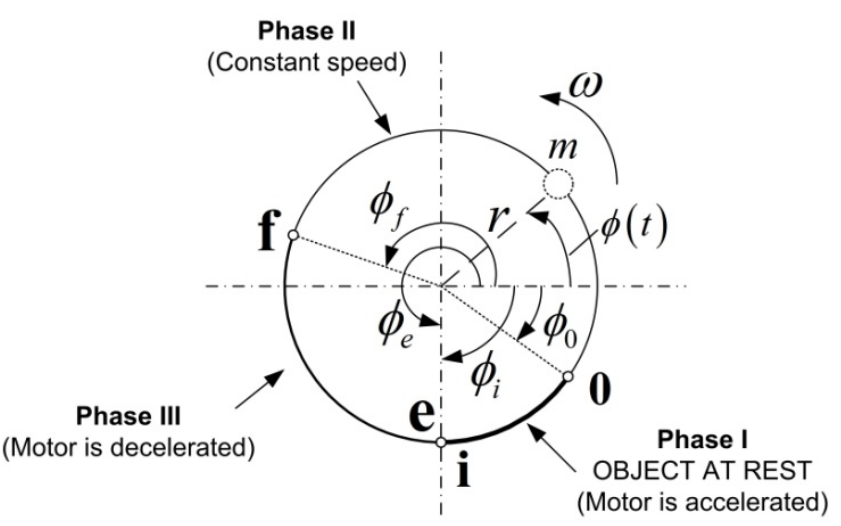

Phase I: Motor is accelerated (object is at rest)

Phase II: Constant speed

Phase III: Motor is decelerated

Figure 2. The cycle of inertial propulsion.

\subsection{Phase I: Motor acceleration}

This phase refers to the part "i0" (thickest line segment) of Fig. 2, in the anti-clockwise direction. For simplicity, we assume that the angular velocity increases linearly with respect to the time $t$; it generally starts from a small value, $\omega_{i}$ at $\left(\phi_{i}, t=t_{i}=0\right)$, and increases to a higher one, $\omega_{0}$ at $\left(\phi_{0}, t=t_{0}\right)$. From the definition of the angular velocity, $\omega=\dot{\phi}$, it is immediately concluded that:

$$
\phi(t)=\phi_{i}+\omega_{i} t+\dot{\omega} / 2 t^{2}
$$

and

$$
\omega(t)=\omega_{i}+\dot{\omega} t .
$$

Eliminating the time $t$ between (15) and (16), one finally obtains:

$$
\omega(t)=\left(\omega_{i}^{2}+2 \dot{\omega}\left(\phi-\phi_{i}\right)\right)^{1 / 2}, \phi_{i} \leq \phi \leq \phi_{0} .
$$

\section{A. Maximum inertial force}

Equation (7) depicts that the maximum value of the inertial force in the $y$-direction appears when the function $f(\phi)=\left(\omega^{2} \sin \phi-\dot{\omega} \cos \phi\right)$ takes its maximum value. 
The latter is found by taking the first derivative over $\phi$, which by virtue of (17) leads to the nonlinear equation:

$$
3 \tan \phi+2\left(\phi-\phi_{i}\right)+\omega_{i}^{2} / \dot{\omega}=0
$$

\section{B. Cancelling the maximum static friction}

In order the object resists sliding, the maximum induced inertial forces, as determined by the combination of (7) with (18), must be lower than the maximum static friction:

$$
\lambda\left(\omega^{2} \sin \phi-\dot{\omega} \cos \phi\right) \leq F_{f} /(2 m+M)
$$

There are generally two positions at which the inertial force becomes equal to the maximum static friction. Considering the normal force given by (13), both of them have to fulfill the nonlinear equation:

$$
\lambda\left(\omega^{2} \sin \phi-\dot{\omega} \cos \phi\right)= \pm \mu g,
$$

The plus sign in (20) corresponds to a limit that should not be exceeded in order to avoid sliding in the undesired backward direction, whereas the minus sign corresponds to the marginal approach of the maximum static friction in the desired forward direction.

Substituting (17) into (20) one obtains:

$$
\left[\omega_{i}^{2}+2 \dot{\omega}\left(\phi-\phi_{i}\right)\right] \sin \phi-\dot{\omega} \cos \phi= \pm \mu g / \lambda \text {, }
$$

which is a nonlinear equation in $\phi$ that can be solved using the standard Newton-Raphson procedure.

\subsection{Phase II: Constant speed}

This phase corresponds to part "Of" of Fig. 2. One criterion to determine the point " $\mathbf{f}$ " at which the constant speed stops retaining its value, is related to the smoothness of the speed between " $f$ " and the ending point " $e$ ". This issue will be better understood when dealing with the numerical example in Section 4.

\subsection{Phase III: Decreasing speed}

This final phase refers to the part "fe" of Fig. 2. Below we shall show a way to achieve a smooth transmission between the two kinematic states, i.e. $\left(\phi_{f}, \omega_{f}, t_{f}\right)$ and $\left(\phi_{e}, \omega_{e}, t_{e}\right)$, in the beginning and at the end of the part "fe", respectively. We are seeking for a smooth variation of the function $\phi(t)$ to bridge the aforementioned states. Assuming $C^{1}$-continuity (i.e. continuity of first derivatives), the variation will be described by a polynomial of third degree:

$$
\phi(\eta)=C_{0}+C_{1} \eta+C_{2} \eta^{2}+C_{3} \eta^{3},
$$

where the normalized variable is given by

$$
\eta=\left(t-t_{f}\right) /\left(t_{e}-t_{f}\right)
$$

The coefficients in (22) are calculated fulfilling the following boundary conditions:

$$
\begin{aligned}
& \phi_{1}=\phi(0)=C_{0} \\
& \omega_{1}=\phi^{\prime}(0)=C_{1} \\
& \phi_{2}=\phi(1)=C_{0}+C_{1}+C_{2}+C_{3} \\
& \omega_{2}=\phi^{\prime}(1)=C_{1}+2 C_{2}+3 C_{3}
\end{aligned}
$$

Solving the above linear system we obtain:

$$
\begin{aligned}
& C_{0}=\phi_{f} \\
& C_{1}=\omega_{f} \\
& C_{2}=\omega_{e}-\phi_{e}+\phi_{f} \\
& C_{3}=2 \phi_{e}-\omega_{e}-2 \phi_{f}-\omega_{f}
\end{aligned}
$$

Substituting (25) into (22) and extracting common factors one finally obtains

$$
\begin{aligned}
\phi(\eta) & =\phi_{f}\left(1-3 \eta^{2}+2 \eta^{3}\right)+\omega_{f}\left(\eta-2 \eta^{2}+\eta^{3}\right) \\
& +\phi_{e}\left(3 \eta^{2}-2 \eta^{3}\right)+\omega_{e}\left(-\eta^{2}+\eta^{3}\right)
\end{aligned}
$$

Based on the normalized expression (26), the variation of the angular velocity is obtained by differentiating (26) over $\eta$ and then multiplying by $d \eta / d t$, which by virtue of (23) finally leads to:

$$
\begin{array}{r}
\omega(\eta)=\frac{1}{\left(t_{2}-t_{1}\right)} \cdot\left[\phi_{f}\left(-6 \eta+6 \eta^{2}\right)+\omega_{f}\left(1-4 \eta+3 \eta^{2}\right)\right. \\
\left.+\phi_{e}\left(6 \eta-6 \eta^{2}\right)+\omega_{e}\left(-2 \eta+3 \eta^{2}\right)\right]
\end{array}
$$

In other words, the proposed variation of the angular velocity according to (27) ensures the smooth transmission from the initial kinematic state, $\left(\phi_{e}, \omega_{e}, t_{e}\right)$, to the final one, $\left(\phi_{f}, \omega_{f}, t_{f}\right)$. The four polynomials involved in (27) are called Hermite polynomials.

\subsection{Forward Travel Per Cycle}

If we can ensure that during a propulsion cycle the object performs a strictly forward motion for the entire time interval $\left[t_{0}, t_{e}\right]$, the problem is linear (monotonic direction of the friction), thus it is possible to integrate (6) with respect to the time $t$, which yields:

$$
\dot{y}_{M}=v_{0}+\lambda \omega_{0}\left(\cos \phi_{0}-\cos \phi\right)-\mu g\left(t-t_{0}\right),
$$

and finally: 


$$
\begin{aligned}
y_{M}= & y_{0}+v_{0}\left(t-t_{0}\right)-1 / 2 \mu g\left(t-t_{0}\right)^{2} \\
& +\left(\lambda \omega_{0} \cos \phi_{0}\right)\left(t-t_{0}\right)-\lambda\left(\sin \phi-\sin \phi_{0}\right),
\end{aligned}
$$

where the subscript ' 0 ' refers to the initial conditions at the point ' 0 ' of Fig. 2 at which the object starts sliding.

The application of (28) for an object with a zero initial and

a zero final velocity at time $t=t_{e}$ (i.e. $v_{0}=0, \phi_{e}=2 \pi+\phi_{i}$ whence $\cos \phi_{e}=0$, and $\dot{y}_{M}=0$ ) leads to a time difference:

$$
t_{e}-t_{0}=\frac{\lambda \omega_{0} \cos \phi_{0}}{\mu g} .
$$

Furthermore, adding to (30) the time interval $t_{0}-t_{i}=\omega_{0} / \dot{\omega}$ which is required for the part (i0) of the cycle (shown in Fig. 2), the entire period of the cycle $\left(T=t_{e}-t_{i}\right)$ becomes:

$$
T=\frac{\lambda \omega_{0} \cos \phi_{0}}{\mu g}+\frac{\omega_{0}}{\dot{\omega}}
$$

Clearly, in the above analysis the object starts at rest $\left(v_{0}=0\right.$ at $t=t_{i}$ ) and completes the cycle again at rest ( $\dot{y}_{M}=0$ at $t=T$ ). It is evident that the above module can be continuously repeated forever, provided the motor consumes energy. It is reminded that the latter (motor) is devoted to guide the eccentric masses according to the previously mentioned three phases (I, II, and III). These three phases constitute what we call predefined angular velocity $\omega(t)$ within the time intervals $[0, T],[T, 2 T]$, and so on.

Finally, substituting (30) into (29), the entire distance $\left(\Delta y=y_{e}-y_{0}\right)$ travelled in every full rotation of period $T$ is given by the following expression of closed form:

$$
\Delta y=\frac{\left(\lambda \omega_{0} \cos \phi_{0}\right)^{2}}{2 \mu g}+\lambda\left(1+\sin \phi_{0}\right)
$$

Based on (31) and (32), the average velocity of locomotion can be calculated using the formula:

$$
\overline{\dot{y}}_{M}=\frac{\Delta y}{T}
$$

\section{Numerical example}

\subsection{Data}

For the mechanical system we consider the following data:

- Rotating mass at the end of rigid rod: $m=1.0 \mathrm{~kg}$

- Mass of the object: $M=5 \cdot 0 \mathrm{~kg}$

- Radius of rigid rod: $r=0 \cdot 10 \mathrm{~m}$
- Acceleration of gravity: $g=9 \cdot 81 \mathrm{~m} / \mathrm{s}^{2}$

- Coefficient of static friction: $\mu_{s}=0.3$

- Coefficient of kinetic friction: $\mu_{k}=0.2$

For the design we take:

- Initial angular velocity: $\omega_{i}=0$

- Initial polar angle: $\phi_{i}=-\pi / 2$

- Final polar angle: $\phi_{e}=3 \pi / 2$

- Initial object's velocity: $v_{0}=0$

\subsection{Parametric Calculations}

In the beginning we present calculations exactly at that point where the maximum static friction is canceled. Figure 3 shows the variation of the resultant inertial force (in $y$-direction) versus the elapsed time. These plots have been obtained considering a standard acceleration of the angular velocity ( $\dot{\omega}=54 \mathrm{~s}^{-1}$ ), for three different coefficients of friction $(\mu=0.3,0.4$, and 0.5$)$, for which the values at which the maximum static friction is overwhelmed are represented by the points F1, F2 and F3. These values have been calculated solving (20) and the numerical results are given in Table 1.

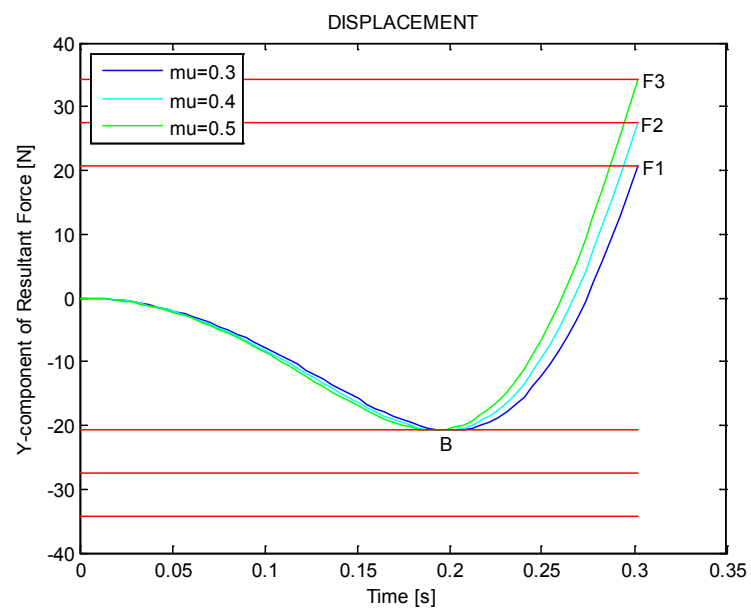

Figure 3. Resultant forces in the $y$-direction compared with the corresponding maximum static forces (shown by the solid red lines) during the time interval $\left[t_{i}, t_{0}\right]$ of every cycle.

In Fig. 3, one can notice two areas, B and F, which refer to backward and forward sliding, respectively. In more detail, the point $B$ is the root of (20) that corresponds to the sign (+) and does not practically depend on the coefficient of friction. In contrast, the points $F(F 1, F 2$ and F3) correspond to the sign (-) of (20) and do depend on the coefficient of friction.

Table 1. Influence of coefficient of friction at the start of object's motion.

\begin{tabular}{|c|c|c|c|}
\hline$\mu$ & $t_{0}[\mathrm{~s}]$ & $\phi_{0}[\mathrm{rad}]$ & $\omega_{0}\left[\mathrm{~s}^{-1}\right]$ \\
\hline 0.3 & 0.2870 & 0.6529 & 15.4971 \\
\hline 0.4 & 0.2945 & 0.7704 & 15.9013 \\
\hline 0.5 & 0.3017 & 0.8871 & 16.2927 \\
\hline
\end{tabular}




\subsection{Calculations for the Entire Cycle}

In this subsection we shall show that it is possible to create a repeated cycle for the anti-clockwise exciting masses, which start rotating at the initial angle $\phi_{i}=-\pi / 2$ and finish at the same position $\left(\phi_{e}=2 \pi+\phi_{i}=3 \pi / 2\right)$ after their rods have kept track on 360 degrees (see, Fig. 2). We shall settle a proper piecewise continuous law for the angular velocity $\omega(t)$ so as to ensure a periodic motion of both the attached rods and the object. According to the data, we have decided to use zero angular velocity $\left(\omega_{i}=0\right)$ and zero object velocity $\left(v_{0}=0\right)$ not only in the beginning of the first period but of every subsequent period. In the sequence we consider that the coefficients of static and kinetic friction take the standard values $\mu_{s}=0.3$ and $\mu_{k}=0.2$, respectively.

\subsubsection{First phase: Speed increase}

In order to create space for increasing the momentum of the rotating masses, we have chosen the initial point of the propulsive cycle to be the angle $\phi_{i}=-\pi / 2$ at the initial time $t_{i}=0$, where the rods are at rest $\left(\omega_{i}=0\right)$ and the object also at rest, i.e. $v_{0}=0$. Starting from this point, and assuming a constant angular acceleration $\dot{\omega}_{i \rightarrow 0} \equiv \dot{\omega}$, the obstacle to avoid is not to exceed the maximum static friction (at an angle $\phi$ determined by (21) in conjunction with plus sign); in the latter case the object would start moving in the opposite to the desired direction (point B in Fig. 3). Having passed this obstacle, (21) in conjunction with minus sign offers the initial angle $\phi_{0}$ at time $t_{i}=t_{0}$ where the object starts sliding. This can be further inserted into (17) to calculate the corresponding speed $\omega_{0}$. For the abovementioned data, it was found that $t_{0}=0.2870 \mathrm{~s}$ and $\phi_{0}=0.6529 \mathrm{rad}$ (=37.4 degrees).

\subsubsection{Second phase: Speed preservation}

As previously explained, during the initial time interval $\left[t_{i}, t_{0}\right)$ of the cycle the object is at rest and "stuck" in the ground, due to the static friction. At the known time $t=t_{0}$ the object starts sliding influenced by the resisting kinetic friction (stick-slip phenomenon). Theoretically, it could be possible to omit the point 'f' of the cycle and select such a variation of the angular velocity $\omega(t)$ from $t=t_{0}$ (point ' $\boldsymbol{0}$ ') until the end of the cycle (point ' $\mathbf{e}$ ') that ensures $C^{1}$-continuity in the function $\phi(t)$. This can be achieved applying (27) in a slightly different manner, which is now between the cycle points ' $\boldsymbol{0}$ ' and ' $\mathbf{e}$ ', instead of the previously mentioned 'f' and 'e'.

However, the shortcoming of this choice is the backward sliding (small but negative displacement $y_{M}$ ) appearing in the beginning of motion, as shown in Fig. 4.

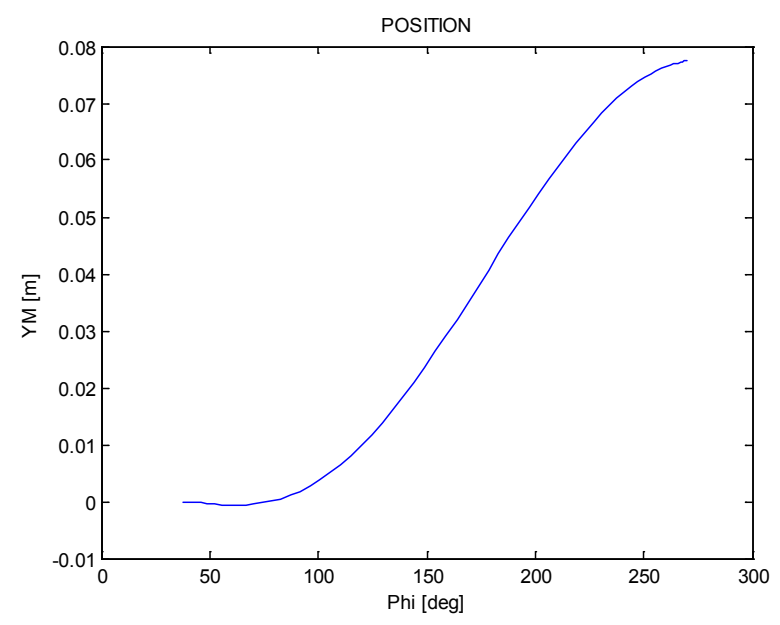

Figure 4. Displacement versus rotation angle using Hermite type variation ( $\omega=\omega_{0}=15.4971 \mathrm{~s}^{-1}, \mu=0.3$ ).

Table 2. Dependence of object forward displacement on the angle $\phi_{f}$.

\begin{tabular}{|c|c|c|c|}
\hline \multirow{2}{*}{$\phi_{f}$} & \multicolumn{2}{|c|}{ At the state $\left(t_{f}, \phi_{f}\right)$} & \multirow{2}{*}{ Remarks } \\
\cline { 2 - 3 }$\left[\begin{array}{c}y_{M} \\
{[\mathrm{~m}]}\end{array}\right.$ & $\begin{array}{c}\dot{y}_{M} \\
{[\mathrm{~m} / \mathrm{s}]}\end{array}$ & \\
\hline 60 & 0.0009 & 0.0804 & \\
\hline 80 & 0.0038 & 0.1807 & \\
\hline 100 & 0.0091 & 0.2903 & \\
\hline 120 & 0.0168 & 0.3906 & \\
\hline 140 & 0.0265 & 0.4642 & \\
\hline 142.6 & 0.0279 & 0.4710 & Symmetrically to point “ 0 ” \\
\hline 160 & 0.0374 & 0.4969 & \\
\hline 180 & 0.0485 & 0.4794 & \\
\hline 200 & 0.0586 & 0.4085 & \\
\hline 220 & 0.0666 & 0.2874 & \\
\hline 240 & 0.0713 & 0.1254 & \\
\hline 260 & 0.0720 & -0.0633 & Backward sliding \\
\hline & & & \\
\hline
\end{tabular}

In order to avoid the above-mentioned backward sliding, it is proposed to preserve the speed $\omega_{0}$ during a time interval $\left[t_{0}, t_{f}\right]$, or equivalently until a certain angle $\phi_{f}$. As shown in Table 2, the selection of $\phi_{f}$ highly influences the corresponding position and velocity of the object at the time instance $t=t_{f}$, for which we remind that $t_{0}<t_{f}<t_{e}$.

\subsubsection{Third phase: Smooth Variation o Speed}

Next it becomes necessary to bridge the gap between the conditions at time $t_{f}$ with those at the end of the period, 
where the end is again that appearing in the beginning of the cycle, i.e. identical with that at time $t_{i}=0$. Since at time $t_{f}$ two quantities $\left(\omega_{f}, \phi_{f}\right)$ are known, while at time $t_{f}$ again two quantities $\left(\omega_{e} \equiv \omega_{i}, \phi_{f} \equiv \phi_{i}+2 \pi\right)$ are known, the gap between them can be easily bridged using cubic Hermitian interpolation, according to (27). The only remaining issue is to determine the period $T=t_{e}-t_{i}$ of the entire cycle, which is predicted using (31).

Based on the findings of Table 2, it is preferable to choose a relatively small value of $\phi_{f}$, and in no case greater than 253 degrees in order to avoid backward sliding. The influence of several typical $\phi_{f}$-values is shown in Fig. 5, while corresponding velocities of the object are shown in Fig. 6. It is noted that when the angle $\phi_{f}$ approaches 196 degrees the velocity of the object exceeds $62 \mathrm{~m} / \mathrm{s}$, while for higher than 197 degrees it becomes negative (backward sliding) and obtains the value $-82 \mathrm{~m} / \mathrm{s}$ between the points " $\mathrm{f}$ " and "e" (not shown). Another numerical finding is that all choices lead to the same maximum distance travelled, a fact that is justified by (32), for which inspection shows that it does not include the parameter $\phi_{f}$. Again, this happens only when no backward sliding occurs. Therefore, for the numerical data of this study, when the $\phi_{f}$ values are higher than 197 degrees, (32) is no more applicable.

Furthermore, in order to better understand the practical point of view, the variation of the motor speed is shown in Fig. 7. Generally, in Phase-III the angular velocity increases from $\omega_{f} \equiv \omega_{0}$ to a certain maximum value and then decreases to zero at $\phi_{e}=3 \pi / 2$. It can be noticed that the smaller the angle $\phi_{f}$ the lower the maximum value of the motor speed is.

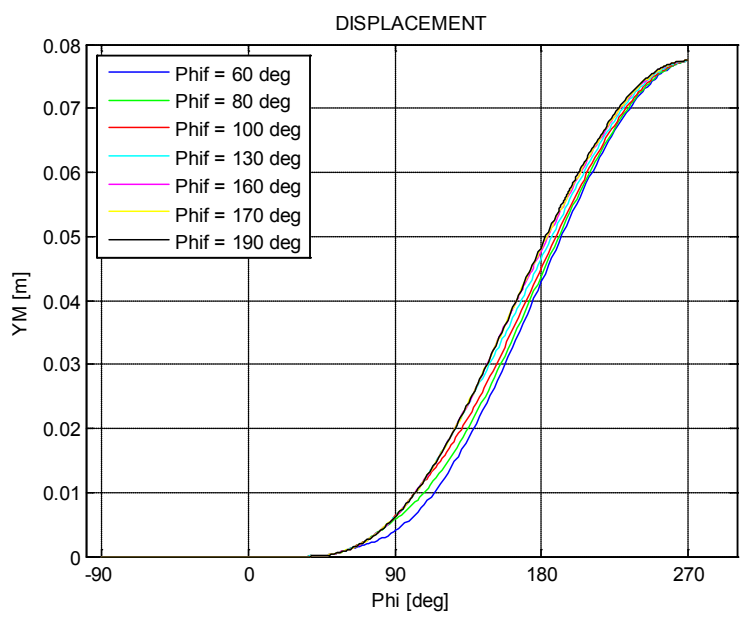

Figure 5. Displacement of the object for several positions $\phi_{f}$ (denoted by Phif) in the beginning of cubic Hermite variation ( $\omega_{f}=\omega_{0}=15.4971 \mathrm{~s}^{-1}, \mu=0.3$ ).

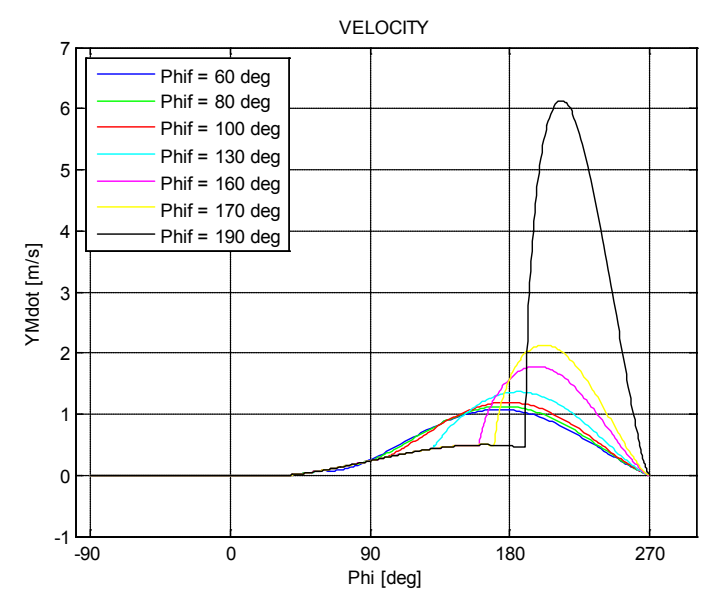

Figure 6. Velocity of the object for several positions $\phi_{f}$ (denoted by Phif) in the beginning of cubic Hermite variation

$\left(\omega_{f}=\omega_{0}=15.4971 \mathrm{~s}^{-1}, \mu=0.3\right)$

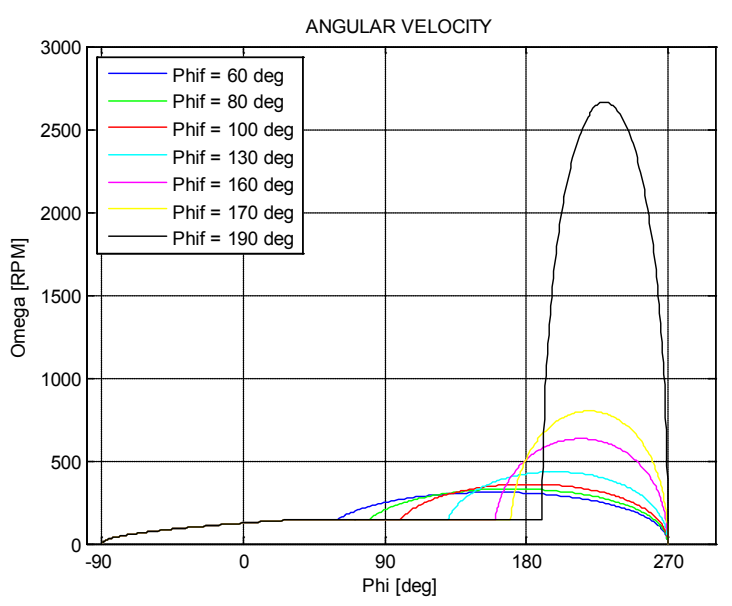

Figure 7. Motor speed (in RPM) for several positions $\phi_{f}$.

\section{Discussion}

An Internet search reveals a great number of publications referring to "reactionless" propulsion, where Newton's third law is supposedly violated ([19], among others). The objective of relevant previous research is concerned with the minimization of energy consumption (maximization of coefficient of efficiency) in machines, in the sense that, the less the reaction the less is the energy consumption. However, the bulk of attempts is also motivated by the need of mankind to perform interstellar journals, in the distant future when the issue of available propellants will be of paramount importance $[8,11,15,16,25,26]$.

Another practical reason that may justify the industrial interest in alternative propulsion means, under certain circumstances, is to avoid exchange of the propulsive machine with the surroundings, for land (no expelling reaction masses) and sea applications (no turbulence). For 
example, it may be desired to navigate the submarine slow motion without rotating its propeller, thus minimizing noise emissions. Although this study was concerned with frictional terrestrial ground, the overall idea can be expanded to motion or/and navigation of autonomous underwater vehicles (AUV) as well. However, in all cases, the restriction is the very low velocity of the object that can be achieved by the proposed inertial means.

The literature survey has shown that, there are very few peer reviewed papers dealing with inertial forces as propulsion means. So far, except of the case of inertial guidance [20] and inertial navigation [21], studies about the above controversial issue to produce propulsion using inertial masses do not seem to have appeared in the public domain. Therefore, they still remain as mysteries of the Internet. In the framework of ground micro-motion, centripetal forces have been previously used to activate microrobotic platforms [4]. More generally, inertial forces have been used to achieve propulsion in micro-/nano-systems [22], control [23], force balancing [24] and space applications [25-27].

With respect to the proposed propulsion cycle there are also some technical remarks to be made. As was mentioned in subsection 4.3.3, during the last part of the cycle, i.e. Phase III, the motor starts from a considerable value $\omega_{f}$ and vanishes to $\omega_{e}=0$. Although we have labeled this phase as "motor deceleration", the truth is that the speed increases at an intermediate value and then vanishes towards $\omega_{e}=0$. It is anticipated that a more detailed design, for example the split of the unique Hermite interpolation in more segments or, probably the uses of B-splines could improve the situation. However such an investigation is out the scope of this paper.

A second remark about the design of the cycle has to be made. It should become clear that it is not generally necessary to impose a zero value of the angular velocity at the beginning of time $\left(t_{i}=0\right)$. Also, it is generally possible to demand for a prescribed nonzero object velocity in the start of every cycle. Nevertheless, the limitation of our proposal is that velocities must be in such a range that transmission from maximum static friction to kinetic friction should be ensured at the beginning and the end of every cycle. Moreover, several other scenarios could be followed for the variation of the rotation during the several subsequent time intervals. No particular attention was paid to reduce energy consumption in the motor or increase the average velocity of the object. Such an optimization is left as a future work. Also, no particular attention was paid to define the limitations in the mass ratio, $m / M$, that can be handled through usual motors.

\section{Conclusion}

It was shown that a device (drive), which consists of motors transmitting torque to contra-rotating masses located at the ends of two rigid bars (eccentrics), when attached to a rigid object is capable of producing terrestrial sliding (locomotion) in any specific direction. The contribution of this research is the finding that, when the aforementioned rods are guided according to a proposed law that predefines the variable angular velocity during one rotation, the device can cause a continuous and repeatable unidirectional motion of the object. The impulsive cycle of this endless sliding is based on the maximum static friction as well as on the kinetic friction. Based on the values of coefficient of static and kinetic friction, we define the variation of the motor speed to be such as it initially ensures the support of the object as long as the motor needs to obtain adequate speed. Then the object starts sliding in a strictly forward way without any backward motion at all, until the mechanical system comes again at rest, after a full rotation of the rods. Thus it continues operating for an innumerous number of cycles, as long as the motor operates. Evidently, the aforementioned motion is performed due to energy consumption by the guiding motors, the actual reaction force is the friction, and no law of mechanics is violated. The overall idea is anticipated to be applicable to sea applications as well.

\section{REFERENCES}

[1] A. E. Minetti, L. P. Ardigo. Halteres used in ancient Olympic long jump, Nature, Vol.420, 141 - 142, 2002.

[2] C. G. Provatidis. Simplified biomechanics for a possible explanation of the Greek long jump using halters, Universal Journal of Engineering Science, Vol. 1, No. 1, 5-16, 2013.

[3] R. A.Thompson, M. F. Wehling, and J. E. Evers. Evaluation of the haltere as a biologically-inspired inertial rate sensor. AIAA Guidance, Navigation, and Control Conference, August 2008, AFRL-RW-EG-TP-2009-7030. (Available at: http://www.dtic.mil/cgi-bin/GetTRDoc?AD=ADA495538)

[4] P. Vartholomeos, E. Papadopoulos. Dynamics, design and simulation of a novel microrobotic platform employing vibration actuators, Journal of Dynamic Systems, Measurement and Control, Transactions of the ASME. Vol. 128, No. 1, 122-133, 2006

[5] C. Zhao, H. Zhu, Y. Zhang, B. Wen. Synchronization of two coupled exciters in a vibrating system of spatial motion, Acta Mech Sin, Vol. 26, 477-493, 2010.

[6] M. Todeschini. Motore a forza propulsive centrifuga alimentato ad acqua dissociate con variazione di velocita graduale automatic. Regno d'Italia, Ministero delle Corporazioni, Brevetto Industriale No. 312496, 17 Novembre 1933; see the first two pages of the patent at the website www.circolotodeschini.com.

[7] N. L. Dean. System for converting rotary motion into unidirectional motion. US Patent 2,886,976, May 19, (1959); also: US Patent 3,182,517, May 11, (1965); $\mathrm{http}: / /$ deanspacedrive.org

[8] M. G. Millis, E. W. Davis (Eds.). Frontiers of Propulsion Science, Progress in Astronautics and Aeronautics Series, Vol. 227. Published by AIAA, Reston, Virginia, 2009. 
[9] I. I. Blekhman. Vibrational Mechanics: Nonlinear dynamic effects, General approach, Applications. World Scientific, Singapore (2000)

[10] G. Y. Stepanov. Why is it impossible to have 'Dean's Apparatus'? Journal Priroda (in Russian), Vol. 7, 85-91, 1963.

[11] (---): Russian scientists test perpetual motion machine in space, PRAVDA Newspaper, 14.04.2009 Accessed on 12th May 2013: http://english.pravda.ru/science/tech/14-04-2009/107399-rus sian_scientists-0/

[12] C. G. Provatidis. Some issues on inertia propulsion mechanisms using two contra-rotating masses, Theory of Mechanisms and Machines, Vol. 8, No. 1, 34-41, 2010.

[13] C. G. Provatidis. A Study of the Mechanics of an Oscillating Mechanism. International Journal of Mechanics, Vol. 5, No. 4, 263-274, 2011.

[14] C. G. Provatidis, M. A. Gamble. Support forces in a synchronized rotating spring-mass system and its electromagnetic equivalent, International Journal of Applied Electromagnetics and Mechanics, Vol. 41, 313-334, 2013.

[15] G. A. Robertson, D. W. Webb. The Death of Rocket Science in the 21st Century, Physics Procedia, Vol. 20, 319-330, 2011.

[16] D. Hambling. EmDrive: China's radical new space drive. Wired Magazine (UK), 06 February (2013) (http://www.wired.co.uk/news/archive/2013-02/06/emdriveand-cold-fusion)

[17] A. B. Engel, P.H. Stiebitz. The cybernetics of inertial Propulsion, Kybernetes, Vol. 38, No. (1/2), 141-157, 2009.

[18] R. F. Dempewolff. Engine with Built-in Wings, Popular Mechanics, Vol. 116, No. 3, 131-134 \& 264-266, 1961.

[19] Selected Internet publications:

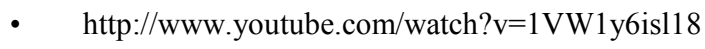

- $\quad$ http://www.youtube.com/watch?v=IeWk5vIEIbo

- http://www.inertialpropulsion.com/

•http://www.americanantigravity.com/tag/inertial-propulsion

- $\quad \mathrm{http}: / / \mathrm{www}$. youtube.com/watch?v=68SiteyZgEo

[20] A. B. Chatfield. Fundamentals of High Accuracy Inertial Guidance. American Institute of Aeronautics and Astronautics, Inc, 1997.

[21] K. R. Britting. Inertial Navigation System Analysis. John Wiley and Sons, Inc, 1971.

[22] H. Yoshikawa, T. Kagiwada, H. Harada, M. Mimura, 2007. Improvement of propulsion mechanism based on the inertial force, in: F. Kimura and K. Horio (Eds.), Towards Synthesis of Micro-/Nano-systems, Springer, London, Proc. 11th International Conference on Precision Engineering (ICPE), August 16-18, 2006, Tokyo, Japan, pp. 333-334.

[23] J. M. Gilbert. Gyrobot: Control of multiple degree of freedom underactuated mechanisms using a gyrating link and cyclic braking, IEEE Transactions on Robotics. 23(4) 822-827, 2007.

[24] P. R. Ouyang, Q. Li, W. J. Zhang, Integrated design of robotic mechanisms for force balancing and trajectory tracking, Mechatronics, Vol. 13, 887-905, 2003.

[25] M. G. Millis, N. E. Thomas. Responding to Mechanical Antigravity, NASA/TM-2006-214390, AIAA-2006-4913, December 2006. Available at:

[26] http://gltrs.grc.nasa.gov/reports/2006/TM-2006-214390.pdf

[27] M. G. Millis. Assessing Potential Propulsion Breakthroughs. In New Trends in Astrodynamics and Applications. ed. Edward Belbruno. New York: Annals of the New York Academy of Sciences (1065), 441-461, 2005 (doi: 10.1196/annals.1370.023).

[28] D. Kouznetsov. Support of non-traditional concepts, Far East Journal of Mechanical Engineering and Physics, Vol. 1, No. 1, $1-6,2010$. 